\title{
Robust Adaptive Tube Model Predictive Control
}

\author{
Xiaonan $\mathrm{Lu}^{*}$
}

Mark Cannon*

\begin{abstract}
An adaptive Model Predictive Control (adaptive MPC) strategy is proposed for linear systems with constant unknown model parameters, bounded additive disturbances and state and control constraints. By combining online set-based identification and robust tube MPC, the proposed controller reduces the conservativeness of constraint handling, guarantees recursive feasibility and provides asymptotic bounds on the closed loop system state that depend explicitly on the the identified parameter set. Computational tractability is ensured by using fixed complexity polytopic sets to bound the model parameters and predicted states. Convex conditions for persistence of excitation are considered. The results are illustrated by a numerical example.
\end{abstract}

\section{INTRODUCTION}

Robust Model Predictive Control (MPC) uses numerical optimization to approximate the solutions of optimal control problems with state and input constraints in the presence of model uncertainty. However, performance depends on model quality since inaccurate models give poor prediction accuracy [1]. As a result, bounds on the achievable performance of a robust MPC strategy are strongly dependent on the amount of uncertainty in the system model [2].

On the other hand, uncertain system models can be improved online using adaptive control. Algorithms combining adaptive control and MPC therefore have the potential to reduce the conservativeness of robust MPC while providing robust stability guarantees. However, although adaptive MPC algorithms have been considered for several decades [3], computational tractability remains an obstacle for implementations of robustly stable adaptive MPC algorithms [4], [5].

Recent developments in adaptive MPC involve different strategies for parameter identification, such as comparison sets [6], set membership identification [7], recursive least squares [8], and neural networks [9], [10]. Specifically, in [6] safety and performance requirements are decoupled by maintaining a nominal model for constraint satisfaction, and a model learnt using statistical identification tools for performance. However, the approach cannot guarantee robust constraint satisfaction and cannot use model adaptation to improve constraint handling. In [7], the system is assumed to have a Finite Impulse Response (FIR) model with constraints and additive disturbances. Set-membership identification techniques are used to determine a set of candidate plants and enforce constraints for all plants in the model. Although recursive feasibility is proven in [7], the FIR assumption is restrictive. Online set-membership identification is used in [11] with a linear state-space model and additive

\footnotetext{
*Department of Engineering Science, University of Oxford, Parks Road, Oxford OX1 3PJ, UK

xiaonan.luleng.ox.ac.uk, mark.cannondeng.ox.ac.uk
}

disturbances. The main disadvantage of the approach is the lack of flexibility in its robust MPC formulation, which is based on homothetic tubes [12], and allows only the centers and scalings of tube cross-sections to be optimized online.

In this paper we also consider state space systems, setbased model identification and robust tube MPC. Compared with [7], [11], the algorithm provides flexibility in modelling the effects of model uncertainty on predicted input and state trajectories, incorporates a convex approximation for persistency of excitation, and gives asymptotic bounds on the closed loop state that depend explicitly on the identified parameter set. In particular, a zonotopic description is used so that the identified parameter set has a pre-specified complexity. This is combined with a tube MPC formulation based on [13] with fixed complexity polytopic sets. As a result the approach retains computational tractability but allows greater flexibility in the definition of predicted state tubes than [11] since the shapes of the tube cross-sections are optimized in addition to their centers and scalings. We propose a convex constraint that ensures a persistence of excitation (PE) condition, and incorporate this in an MPC algorithm with guaranteed stability. The PE condition facilitates convergence of the identified model to the true system parameters, thus extending the result of [14] to MIMO systems. Finally, we show that the resulting MPC strategy is recursively feasible and give asymptotic bounds on the convergence of the closed loop state that depend on the identified parameter set. This bounding set is used in the definition of the MPC cost and is updated online as the parameter set estimate is updated.

Notation: The sets of integers and reals are denoted $\mathbb{N}$ and $\mathbb{R}$ respectively, and we define $\mathbb{N}_{\geq 0}=\{n \in \mathbb{N}: n \geq 0\}$, $\mathbb{N}_{[a, b]}=\{n \in \mathbb{N}: a \leq n \leq b\}$. The $i$ th row of a matrix $A$ and $i$ th element of a vector $a$ are $[A]_{i}$ and $[a]_{i}$ respectively. Vectors and matrices of $1 \mathrm{~s}$ are denoted $\mathbf{1}$, and $\mathbb{I}$ represents the identity matrix. For any real scalar-valued function $J$, $\max _{x \in \mathbb{X}} J(x)$ means the maximum value of $J$ over the set $\mathbb{X}$. For a vector $a, \max a$ means the maximum element of the vector $a$, and for scalar $x$ we define $[x]_{\geq 0}=\max \{0, x\}$. The inequality $A \geq B$ applies elementwise, while $A \succeq B$ for square matrices $A, B$ means $x^{\top} A x \geq x^{\top} B x$ for all vectors $x$ of conformal dimensions. The $k$-steps ahead predicted value of a variable $z$ is denoted $z_{k}$, and the more complete notation $z_{k \mid t}$ indicates the $k$-steps ahead prediction at time $t$.

\section{PROBLEM FORMULATION AND PRELIMINARIES}

This paper considers a linear system with linear state and input constraints and unknown additive disturbance:

$$
x_{t+1}=A(\theta) x_{t}+B(\theta) u_{t}+w_{t},
$$


Here $x_{t} \in \mathbb{R}^{n_{x}}$ is the system state, $u_{t} \in \mathbb{R}^{n_{u}}$ is the control input, $w_{t} \in \mathbb{R}^{n_{x}}$ is the disturbance input, and $t$ is the discrete time index. The state and input constraints are given by

$$
F x_{t}+G u_{t} \leq \mathbf{1} \quad \forall t \in \mathbb{N}_{\geq 0}
$$

for given $F \in \mathbb{R}^{n_{c} \times n_{x}}$ and $G \in \mathbb{R}^{n_{c} \times n_{u}}$. The system matrices $A(\theta)$ and $B(\theta)$ depend on an unknown but constant parameter $\theta \in \mathbb{R}^{p}$.

Assumption 1 (Additive disturbance): the disturbance $w_{t}$ lies in a convex and compact polytope $\mathbb{W}$, where

$$
\mathbb{W}=\left\{w: \Pi_{w} w \leq \pi_{w}\right\}
$$

with $\Pi_{w} \in \mathbb{R}^{q_{w} \times n_{x}}, \pi_{w} \in \mathbb{R}^{q_{w}}$ and $\pi_{w}>0$.

Assumption 2 (Parameter uncertainty): the system matrices are affine functions of the parameter vector $\theta \in \mathbb{R}^{p}$

$$
(A(\theta), B(\theta))=\left(A_{0}, B_{0}\right)+\sum_{i=1}^{p}\left(A_{i}, B_{i}\right)[\theta]_{i}
$$

for known matrices $A_{j}, B_{j}, j=1, \ldots, p$, and $\theta$ lies in a known, bounded, convex polytope $\Theta_{0}$ given by

$$
\begin{array}{ll}
\text { H-form: } & \Theta_{0}=\left\{\theta: \Pi_{\theta} \theta \leq \pi_{0}\right\} \\
\text { V-form: } & \Theta_{0}=\operatorname{co}\left\{\theta_{0}^{(1)}, \ldots, \theta_{0}^{(m)}\right\} .
\end{array}
$$

In order to restrict computational complexity, $\Theta_{0}$ is assumed to adopt the form of zonotope, i.e. $\Pi_{\theta}^{\top}=\left[\Pi^{\top}-\Pi^{\top}\right]$, where $\Pi \in \mathbb{R}^{p \times p}$ is a full-rank matrix. This zonotopic structure allows $\mathrm{H}$ - and $\mathrm{V}$-forms to be easily interchangeable.

To obtain finite numbers of decision variables and constraints in the MPC optimization problem, the predicted control sequences are assumed to have the dual mode form:

$$
u_{k \mid t}= \begin{cases}K x_{k \mid t}+v_{k \mid t} & \forall k \in \mathbb{N}_{[0, N-1]} \\ K x_{k \mid t} & \forall k \geq N\end{cases}
$$

where $v_{0 \mid t}, v_{1 \mid t} \ldots, v_{N-1 \mid t}$ are optimization variables at time $t$ and $N$ is the MPC prediction horizon. The feedback gain $K$ is designed offline and is assumed to robustly stabilize the uncertain system $x_{t+1}=(A(\theta)+B(\theta) K) x_{t}, \theta \in \Theta_{0}$ in the absence of constraints.

Assumption 3 (Feedback gain and contractive set): there exists a polytopic set $\mathbb{X}=\{x: V x \leq \mathbf{1}\}$ and feedback gain $K$ such that $\mathbb{X}$ is $\lambda$-contractive for some $\lambda \in[0,1)$, i.e.

$$
\begin{aligned}
& V(A(\theta)+B(\theta) K) x \leq \lambda \mathbf{1} \\
& \quad \text { for all } x \in\{x: V x \leq \mathbf{1}\} \text { and } \theta \in \Theta_{0} .
\end{aligned}
$$

Given knowledge of $\mathbb{W}$ and an initial parameter set $\Theta_{0}$, the control objective is to solve a robust optimal regulation problem while respecting the constraints (2) and ensuring closed loop stability. Simultaneously with controlling the system, an additional objective is to update the model parameter set $\Theta_{t}$ online using measurements of the state $x_{t}$, and to use the updated parameter set to improve controller performance.

\section{AdAptive Robust MPC}

In this section a suitable parameter estimation scheme based on [15], [16] is introduced. This is followed by the construction of a tube bounding predicted model states and corresponding constraints. Subsequently, the MPC cost is discussed, linearized sufficient conditions for persistent excitation are described, and a summary of the MPC algorithm is given. The section concludes with a summary of closed loop system properties. For brevity, proofs of the various results are omitted, but standard results on polyhedral set inclusion conditions (e.g. [17]) are employed, and we make use of the following result on linearly parameterised sets.

Lemma 4: Let $\mathbb{P}_{1}=\left\{x: F_{1} x \leq f_{1}\right\}$ and $\mathbb{P}_{2}(\theta)=\{x:$ $\left.F_{2}(\theta) x \leq f_{2}\right\}$, where $F_{1} \in \mathbb{R}^{n_{1} \times n_{x}}, F_{2}(\theta) \in \mathbb{R}^{n_{2} \times n_{x}}$ and $\left[F_{2}(\theta)\right]_{i}=\theta^{\top} \hat{F}_{2, i}, i \in \mathbb{N}_{\left[1, n_{2}\right]}$. Then $\mathbb{P}_{1} \subseteq \mathbb{P}_{2}(\theta)$ for all $\theta \in \Theta \subset \mathbb{R}^{p}$ if and only if there exists $\hat{H}_{i} \in \mathbb{R}^{p \times n_{1}}$ satisfying

$$
\theta^{\top} \hat{H}_{i} \geq 0 \quad \hat{H}_{i} F_{1}=\hat{F}_{2, i} \quad \theta^{\top} \hat{H}_{i} f_{1} \leq f_{2, i}
$$

for all $i \in \mathbb{N}_{\left[1, n_{2}\right]}$ and all $\theta \in \Theta$.

\section{A. Parameter estimation}

At time step $t$ we use observations of the system state $x_{t}$, which is assumed to be known, to determine a polytopic set $\Delta_{t}$ of possible model parameters. This unfalsified parameter set is then combined with the current polytopic parameter set estimate to construct a new parameter set estimate $\Theta_{t}$.

1) Unfalsified parameter set: Define $D(x, u) \in \mathbb{R}^{n_{x} \times p}$ as

$$
D(x, u)=\left[\begin{array}{lll}
A_{1} x+B_{1} u & \cdots & A_{p} x+B_{p} u
\end{array}\right],
$$

and let $d_{t} \in \mathbb{R}^{n_{x}}$ be defined

$$
d_{t}=A_{0} x_{t-1}+B_{0} u_{t-1}-x_{t} .
$$

From the disturbance set $\mathbb{W}$ in (3), given $x_{t}, x_{t-1}$ and $u_{t-1}$, the unfalsified parameter set at time $t$ is given by

$$
\begin{aligned}
\Delta_{t} & =\left\{\theta: x_{t}-\left(A(\theta) x_{t-1}+B(\theta) u_{t-1}\right) \in \mathbb{W}\right\} \\
& =\left\{\theta:-\Pi_{w} D\left(x_{t-1}, u_{t-1}\right) \theta \leq \pi_{w}+\Pi_{w} d_{t}\right\} \\
& =\left\{\theta: P_{t} \theta \leq Q_{t}\right\}
\end{aligned}
$$

with $P_{t}=-\Pi_{w} D\left(x_{t-1}, u_{t-1}\right)$ and $Q_{t}=\pi_{w}+\Pi_{w} d_{t}$.

2) Parameter set update: Let $\Pi_{\theta} \in \mathbb{R}^{2 p \times p}$ be an a priori chosen matrix defining the initial parameter set $\Theta_{0}=\{\theta$ : $\left.\Pi_{\theta} \theta \leq \pi_{0}\right\}$. At each time step $t \in \mathbb{N}_{\geq 0}$ the estimated parameter set is defined as $\Theta_{t}=\left\{\theta: \Pi_{\theta} \theta \leq \pi_{t}\right\}$, where $\pi_{t}$ is determined online. The complexity of $\Theta_{t}$ is controlled by fixing $\Pi_{\theta}$, which is equivalent to fixing the directions of the half-spaces defining $\Theta_{t}$. The smallest set $\Theta_{t+1}$ satisfying $\Theta_{t+1} \supseteq \Theta_{t} \cap \Delta_{t}$ is obtained by solving, for each $i \in \mathbb{N}_{[1,2 p]}$ the linear program (LP):

$$
\begin{aligned}
& {\left[\pi_{t+1}\right]_{i}=\min _{\pi \in \mathbb{R}, H_{i} \in \mathbb{R}^{1 \times\left(2 p+q_{w}\right)}} \pi} \\
& \text { s.t. } H_{i}\left[\begin{array}{c}
\Pi_{\theta} \\
P_{t}
\end{array}\right]=\left[\Pi_{\theta}\right]_{i}, H_{i}\left[\begin{array}{c}
\pi_{t} \\
Q_{t}
\end{array}\right] \leq \pi, H_{i} \geq 0 .
\end{aligned}
$$

Lemma 5: If $\Pi_{\theta} \theta \leq \pi_{0}$ and $\pi_{t+1}$ is defined by (6) for all $t \in \mathbb{N}_{\geq 0}$, then $\theta \in \Theta_{t}$ and

$$
\Theta_{t} \supseteq \Theta_{t+1} \supseteq \Theta_{t} \cap \Delta_{t}
$$


for all $t \in \mathbb{N}_{\geq 0}$. Furthermore $\pi_{t+1}$ defines the minimum volume set $\Theta_{t+1}=\left\{\theta: \Pi_{\theta} \theta \leq \pi_{t+1}\right\}$ containing $\Theta_{t} \cap \Delta_{t}$.

Remark 6: Instead of using only the current observation $\Delta_{t}$ to update the parameter set estimate, a tighter set estimate can be achieved at the expense of increased computation by block-processing all past observations or observations in a moving a window of length $n$, see [15] for details.

\section{B. Polytopic tubes for constraint satisfaction}

This section is concerned with predicted state and input trajectories. To simplify notation, we omit the subscript $t$ indicating the time at which predictions are made; in particular the current state $x_{t}$ and parameter set $\Theta_{t}$ are denoted $x, \Theta$, and $k$ steps-ahead predictions of $x$ and $u$ are denoted $x_{k}, u_{k}$. For given $V \in \mathbb{R}^{n_{\alpha} \times n_{x}}$ satisfying Assumption 3 and $\alpha_{k} \in \mathbb{R}^{n_{\alpha}}$, let

$$
\mathbb{X}_{k}=\left\{x: V x \leq \alpha_{k}\right\}
$$

To ensure that the predicted state and control sequences satisfy the operating constraints (2) robustly for the given uncertainty bounds we construct a tube (sequence of sets) $\mathbb{X}_{0}, \mathbb{X}_{1}, \ldots$ satisfying, for all $k \in \mathbb{N}_{\geq 0}$,

$$
\begin{aligned}
& \Phi(\theta) x+B(\theta) v_{k}+w \in \mathbb{X}_{k+1} \\
& \text { for all } x \in \mathbb{X}_{k}, w \in \mathbb{W}, \theta \in \Theta=\operatorname{co}\left\{\theta^{(1)}, \ldots \theta^{(m)}\right\}
\end{aligned}
$$

where $\Phi(\theta)=A(\theta)+B(\theta) K=\sum_{i=0}^{p} \Phi_{i}[\theta]_{i}$ with $\Phi_{i}=$ $A_{i}+B_{i} K$ and $[\theta]_{0}=1$. For notational convenience we also define $\hat{\theta}^{(j)}$ as $\hat{\theta}^{(j) \top}=\left[\begin{array}{ll}1 & \theta^{(j) \top}\end{array}\right]$ for all $j \in \mathbb{N}_{[1, m]}$.

Lemma 7: For $x_{k} \in \mathbb{X}_{k}$ and $u_{k}=K x_{k}+v_{k}$, the constraint (2) is satisfied at the $k$ th prediction time step if

$$
H_{c} \alpha_{k}+G v_{k} \leq \mathbf{1}
$$

where $H_{c}$ is computed offline by solving the LP:

$$
\left[H_{c}\right]_{i}=\underset{H \in \mathbb{R}^{1 \times n_{\alpha}}}{\operatorname{argmin}} H \mathbf{1} \text { s.t. } H \geq 0, H V=[F+G K]_{i} .
$$

for each $i \in \mathbb{N}_{\left[1, n_{c}\right]}$.

Lemma 8: The predicted state tube satisfies (7) if, for all $j \in \mathbb{N}_{[1, m]}$ and $i \in \mathbb{N}_{\left[1, n_{\alpha}\right]}$,

$$
\hat{\theta}^{(j) \top} \hat{H}_{i} \alpha_{k}+[V]_{i} B\left(\theta^{(j)}\right) v_{k}+\bar{w}_{i} \leq\left[\alpha_{k+1}\right]_{i},
$$

where $\bar{w}_{i}=\max _{w \in \mathbb{W}}[V]_{i} w$ and the matrices $\hat{H}_{i}$ are computed offline by solving an LP for each $i \in \mathbb{N}_{\left[1, n_{\alpha}\right]}$ :

$$
\begin{gathered}
\hat{H}_{i}=\underset{H \in \mathbb{R}^{(p+1) \times n_{\alpha}}}{\operatorname{argmin}} \max _{j \in \mathbb{N}_{[1, m]}} \hat{\theta}^{(j) \top} H \mathbf{1} \\
\text { subject to } \hat{\theta}^{(j) \top} H \geq 0, j \in \mathbb{N}_{[1, m]}, \\
H V=\left[\begin{array}{c}
{[V]_{i} \Phi_{0}} \\
\vdots \\
{[V]_{i} \Phi_{p}}
\end{array}\right] .
\end{gathered}
$$

Initial and final conditions on state tubes are imposed as

$$
\begin{aligned}
\alpha_{0} & \geq V x, \\
{\left[\alpha_{N}\right]_{i} } & \geq \hat{\theta}^{(j) \top} \hat{H}_{i} \alpha_{N}+\bar{w}_{i}, \quad \forall j \in \mathbb{N}_{[1, m]}, i \in \mathbb{N}_{\left[1, n_{\alpha}\right]} \\
H_{c} \alpha_{N} & \leq \mathbf{1}
\end{aligned}
$$

Here (12) implies $x \in \mathbb{X}_{0}$ whereas (13) and (14) ensure $\mathbb{X}_{N}$ is robustly invariant for the system $x_{k+1}=\Phi(\theta) x_{k}+w_{k}$ with constraint $F x_{k}+G u_{k} \leq \mathbf{1}$, for all $\theta \in \Theta$ and $w_{k} \in \mathbb{W}$.

Remark 9: The existence of some $\alpha_{N}$ satisfying (13) is ensured by the $\lambda$-contractivity condition (4), which implies that the solution of (11) satisfies $\hat{\theta}^{(j) \top} \hat{H}_{i} \mathbf{1} \leq \lambda$ for all $i \in$ $\mathbb{N}_{\left[1, n_{\alpha}\right]}$. Therefore, with $\alpha_{N}=r \mathbf{1}$ the RHS of (13) has upper bound $\lambda r \mathbf{1}+\bar{w}$, and since $\lambda \in[0,1)$, this choice of $\alpha_{N}$ is feasible for (13) if $r$ is sufficiently large.

\section{MPC Objective function}

Consider a min-max MPC strategy based on a piecewiselinear worst case cost index of the form

$$
\max _{x_{k} \in \mathbb{X}_{k}, k=0,1, \ldots} \sum_{k=0}^{\infty}\left(\max \left\{Q x_{k}\right\}+\max \left\{R u_{k}\right\}\right)
$$

where $Q \in \mathbb{R}^{n_{Q} \times n_{x}}$ and $R \in \mathbb{R}^{n_{R} \times n_{u}}$ are weighting matrices and $\max \{Q x\}$ and $\max \{R u\}$ denote the maximum elements of $Q x$ and $R u$ respectively. We assume that $Q$ and $R$ are chosen so that $\max \{Q x\}$ and $\max \{R u\}$ are positive definite functions of $x$ and $u$, so that $\{x: Q x \leq 1\}$ and $\{u: R u \leq \mathbf{1}\}$ are compact polytopic sets.

Lemma 10: If $s_{k}$ and $t_{k}$ satisfy the linear constraints:

$$
\begin{aligned}
& s_{k} \mathbf{1} \geq H_{Q} \alpha_{k} \\
& t_{k} \mathbf{1} \geq H_{R} \alpha_{k}+R v_{k}
\end{aligned}
$$

where $H_{Q}, H_{R}$ are computed offline by solving the LPs:

$$
\begin{aligned}
& {\left[H_{Q}\right]_{i}=\underset{H \in \mathbb{R}^{1 \times n_{V}}}{\operatorname{argmin}} H \mathbf{1} \text { s.t. } H \geq 0, H V=[Q]_{i}} \\
& {\left[H_{R}\right]_{j}=\underset{H \in \mathbb{R}^{1 \times n_{V}}}{\operatorname{argmin}} H \mathbf{1} \text { s.t. } H \geq 0, H V=[R]_{j} K}
\end{aligned}
$$

for $i \in \mathbb{N}_{\left[1, n_{Q}\right]}$ and $j \in \mathbb{N}_{\left[1, n_{R}\right]}$, then the terms in (15) satisfy

$$
\max _{x \in \mathbb{X}_{k}} \max \{Q x\} \leq s_{k}, \quad \max _{x \in \mathbb{X}_{k}} \max \left\{R\left(K x_{k}+v_{k}\right)\right\} \leq t_{k} .
$$

By Lemma 10, the piecewise-linear worst case cost (15) is bounded by $\sum_{k=0}^{\infty}\left(s_{k}+t_{k}\right)$. However, to avoid the difficulty that this sum is in general infinite because of the presence of persistent additive disturbances, we redefine the cost by penalizing only the amounts that $s_{k}$ and $t_{k}$ exceed upper bounds on asymptotic limits as $k \rightarrow \infty$. The required asymptotic bounds and an associated terminal penalty term are provided by Lemmas 11 and 12, which are based on the stabilizing feedback $K$ and $\lambda$-contractivity condition (4).

Lemma 11: For $k \geq N$, we have $v_{k}=0, u_{k}=K x_{k}$ and

$$
\max _{x \in \mathbb{X}_{k}} \max \{Q x\} \leq \bar{s}_{k}(\lambda), \quad \max _{x \in \mathbb{X}_{k}} \max \{R K x\} \leq \bar{t}_{k}(\lambda)
$$

where $\bar{s}_{k}(\lambda)=\max \left\{H_{Q} \mathbf{1}\right\} \lambda^{k-N} \max \left\{\alpha_{N}\right\}$

$$
\begin{aligned}
& +\frac{1-\lambda^{k-N}}{1-\lambda} \max \left\{H_{Q} \mathbf{1}\right\} \max \{\bar{w}\}, \\
\bar{t}_{k}(\lambda)= & \max \left\{H_{R} \mathbf{1}\right\} \lambda^{k-N} \max \left\{\alpha_{N}\right\} \\
& +\frac{1-\lambda^{k-N}}{1-\lambda} \max \left\{H_{R} \mathbf{1}\right\} \max \{\bar{w}\} .
\end{aligned}
$$

Since $\lambda \in[0,1)$, Lemma 11 implies the asymptotic bounds

$$
\lim _{k \rightarrow \infty} \bar{s}_{k}(\lambda)=\bar{s}_{\infty}(\lambda)=\frac{1}{1-\lambda} \max \left\{H_{Q} \mathbf{1}\right\} \max \{\bar{w}\}
$$




$$
\lim _{k \rightarrow \infty} \bar{t}_{k}(\lambda)=\bar{t}_{\infty}(\lambda)=\frac{1}{1-\lambda} \max \left\{H_{R} \mathbf{1}\right\} \max \{\bar{w}\}
$$

and it follows that $\bar{s}_{\infty}(\lambda)+\bar{t}_{\infty}(\lambda)$ provides an upper bound on the value of $\max \{Q x\}+\max \{R u\}$ for all $x$ in the minimal robust positively invariant set [2] for the uncertain system (1) with $\theta \in \Theta$ under $u=K x$. The proposed MPC algorithm updates this bound as the estimated parameter set $\Theta$ is updated online. Furthermore, convergence of the closed loop system state to a set on which $\max \{Q x\}+$ $\max \{R u\} \leq \bar{s}_{\infty}(\lambda)+\bar{t}_{\infty}(\lambda)$ is ensured (as demonstrated in Proposition 19) by defining the MPC cost as the amount by which $s_{k}+t_{k}$ exceeds this bound. We therefore redefine the MPC cost as

$$
\max _{x_{k} \in \mathbb{X}_{k}, k=0,1, \ldots} \sum_{k=0}^{\infty}\left(\sigma_{k}+\tau_{k}\right)
$$

where $\sigma_{k}, \tau_{k}$ are the amounts by which $s_{k}$ and $t_{k}$ exceed the limits $\bar{s}_{\infty}(\lambda)$ and $\bar{t}_{\infty}(\lambda)$ :

$$
\sigma_{k}=\left[s_{k}-\bar{s}_{\infty}(\lambda)\right]_{\geq 0} \quad \tau_{k}=\left[t_{k}-\bar{t}_{\infty}(\lambda)\right]_{\geq 0}
$$

(with $[s]_{\geq 0}$ denoting $\max \{0, s\}$ for any scalar $s$ ).

Lemma 12: The value of $\sum_{k=N}^{\infty}\left(\sigma_{k}+\tau_{k}\right)$ is bounded by $\bar{\sigma}_{N}+\bar{\tau}_{N}$, where

$$
\begin{aligned}
& \bar{\sigma}_{N}=\left[\max \left\{H_{Q} \mathbf{1}\right\} \frac{1}{1-\lambda}\left(\max \left\{\alpha_{N}\right\}-\frac{1}{1-\lambda} \max \{\bar{w}\}\right)\right]_{\geq 0} \\
& \bar{\tau}_{N}=\left[\max \left\{H_{R} \mathbf{1}\right\} \frac{1}{1-\lambda}\left(\max \left\{\alpha_{N}\right\}-\frac{1}{1-\lambda} \max \{\bar{w}\}\right)\right]_{\geq 0} .
\end{aligned}
$$

Remark 13: From (22), (23) and Lemma 12, the MPC cost function can be expressed

$$
J(x, \mathbf{v}, \boldsymbol{\alpha}, \boldsymbol{\sigma}, \boldsymbol{\tau})=\sum_{k=0}^{N-1}\left(\sigma_{k}+\tau_{k}\right)+\bar{\sigma}_{N}+\bar{\tau}_{N}
$$

where $\mathbf{v}=\left\{v_{0}, \ldots, v_{N-1}\right\}, \boldsymbol{\alpha}=\left\{\alpha_{0}, \ldots, \alpha_{N}\right\}, \boldsymbol{\sigma}=$ $\left\{\sigma_{0}, \ldots, \sigma_{N-1}, \bar{\sigma}_{N}\right\}, \boldsymbol{\tau}=\left\{\tau_{0}, \ldots, \tau_{N-1}, \bar{\tau}_{N}\right\}$ are optimization variables, with $\sigma, \tau$ defined by the linear constraints

$\sigma_{k} \geq 0, \sigma_{k} \mathbf{1} \geq H_{Q} \alpha_{k}-\bar{s}_{\infty}(\lambda) \mathbf{1}$

$\tau_{k} \geq 0, \quad \tau_{k} \mathbf{1} \geq H_{R} \alpha_{k}+R v_{k}-\bar{t}_{\infty}(\lambda) \mathbf{1}$

$\bar{\sigma}_{N} \geq 0, \bar{\sigma}_{N} \geq \frac{1}{1-\lambda}\left(\max \left\{H_{Q} \mathbf{1}\right\} \max \left\{\alpha_{N}\right\}-\bar{s}_{\infty}(\lambda)\right)$

$\bar{\tau}_{N} \geq 0, \bar{\tau}_{N} \geq \frac{1}{1-\lambda}\left(\max \left\{H_{R} \mathbf{1}\right\} \max \left\{\alpha_{N}\right\}-\bar{t}_{\infty}(\lambda)\right)$.

\section{Persistent Excitation}

In [18] it is shown that, if the disturbance bound $w_{t} \in \mathbb{W}$ is tight and the regressor is persistently exciting, then the diameter of the parameter set estimated using set membership identification will converge asymptotically to zero with probability one. In the current context, given a suitable window length, $n$, the PE condition over time steps $t-n+1, \ldots, t$, where $t$ is the current time, is

$$
\sum_{k=t-n+1}^{t} D_{k}^{\top} D_{k} \succeq n \beta_{1}^{2} \mathbb{I}
$$

where $D_{k}=D\left(x_{k}, u_{k}\right)$. This condition is in general nonconvex in $x$ and $u$, and we therefore derive a convex sufficient condition for (29) as follows.
Assume a control input, $u_{0 \mid t}^{o}$, that satisfies the state and input constraints has been obtained at time $t$. To obtain an input $u_{t}$ that additionally satisfies the PE constraint (29), consider applying a perturbation, $\delta u$, to $u_{0 \mid t}^{o}$ :

$$
u_{t}=u_{0 \mid t}^{o}+\delta u \text {. }
$$

Since $D_{t}=D\left(x_{t}, u_{t}\right)$ depends linearly on $\delta u$, we have

$$
\begin{aligned}
\sum_{k=t-n+1}^{t} D_{k}^{\top} D_{k}= & M_{t}+L(\delta u)^{\top} D\left(x_{t}, u_{0 \mid t}^{o}\right) \\
& +D\left(x_{t}, u_{0 \mid t}^{o}\right)^{\top} L(\delta u)+L(\delta u)^{\top} L(\delta u)
\end{aligned}
$$

where $M_{t}$ is independent of $\delta u$,

$$
M_{t}=\sum_{k=t-n+1}^{t-1} D_{k}^{\top} D_{k}+D\left(x_{t}, u_{0 \mid t}^{o}\right)^{\top} D\left(x_{t}, u_{0 \mid t}^{o}\right),
$$

and $L(\delta u)$ is linear in $\delta u$

$$
L(\delta u)=\left[\begin{array}{lll}
B_{1} \delta u & \cdots & B_{p} \delta u
\end{array}\right] .
$$

The quadratic term $L(\delta u)^{\top} L(\delta u)$ is necessarily positive semidefinite and a sufficient condition for (29) is therefore

$$
M_{t}+D\left(x_{t}, u_{0 \mid t}^{o}\right)^{\top} L(\delta u)+L(\delta u)^{\top} D\left(x_{t}, u_{0 \mid t}^{o}\right) \succeq n \beta_{1}^{2} \mathbb{I} .
$$

This is a linear matrix inequality (LMI) constraint on $\delta u$ which is necessarily convex in $\delta u$ and which ensures the PE condition (29) with a degree of conservativeness that depends on $\delta u$ as $O\left(\|\delta u\|^{2}\right)$.

Remark 14: The only variable in (30) is a perturbation $\delta u$ associated with the current time step, and there may not exist a solution that satisfies this condition simultaneously with the constraints. On the other hand, it is not necessary for condition (29), and by implication (30), to be satisfied at every time step $t$ for convergence of the parameter set $\Theta_{t}$.

\section{E. Proposed Algorithm}

Algorithm 1 (Robust Adaptive MPC):

Offline, given an initial parameter set estimate $\Theta_{0}$ :

Choose $V$ and compute $\lambda \in[0,1), K$ and

$$
\begin{array}{ll}
H_{c}, \hat{H}_{1}, \ldots, \hat{H}_{n_{\alpha}} & \text { by solving (9), (11) } \\
H_{Q}, H_{R} & \text { by solving (18), (19). }
\end{array}
$$

Online at times $t \in \mathbb{N}_{\geq 0}$, given the state measurement $x_{t}$ :

1) Update $\Theta_{t}$ using the parameter set identification algorithm (6) and compute its vertices $\theta_{t}^{(1)} \ldots \theta_{t}^{(m)}$.

2) Update $\lambda_{t}$ by solving

$$
\lambda_{t}=\max _{j \in \mathbb{N}_{[1, m]}, i \in \mathbb{N}_{\left[1, n_{\alpha}\right]}} \hat{\theta}_{t}^{(j) \top} \hat{H}_{i} \mathbf{1} .
$$

3) Compute the optimal solution: $\mathbf{v}_{t}=\left\{v_{0 \mid t}, \ldots, v_{N-1 \mid t}\right\}$, $\boldsymbol{\alpha}_{t}=\left\{\alpha_{0 \mid t}, \ldots, \alpha_{N \mid t}\right\}, \boldsymbol{\sigma}_{t}=\left\{\sigma_{0 \mid t}, \ldots, \sigma_{N-1 \mid t}, \bar{\sigma}_{N \mid t}\right\}$, $\tau_{t}=\left\{\tau_{0 \mid t}, \ldots, \tau_{N-1 \mid t}, \bar{\tau}_{N \mid t}\right\}$ of the LP

$$
J^{o}\left(x_{t}\right)=\min _{\mathbf{v}, \boldsymbol{\alpha}, \boldsymbol{\sigma}, \boldsymbol{\tau}} \sum_{k=0}^{N-1}\left(\sigma_{k}+\tau_{k}\right)+\bar{\sigma}_{N}+\bar{\tau}_{N}
$$

subject to (8), (10), (25), (26) $\forall k \in \mathbb{N}_{[0, N-1]}$

$$
\text { (12), (13), (14), (27), (28). }
$$


4) Calculate the control law $u_{0 \mid t}^{o}=K x_{t}+v_{0 \mid t}$ and check the PE condition (29) with $D_{t}=D\left(x_{t}, u_{0 \mid t}^{o}\right)$.

(a) If (29) is satisfied, implement $u_{t}=u_{0 \mid t}^{o}$

(b) Otherwise, recompute the optimization (31) with an additional optimization variable $\delta u$ and with the convex PE condition (30), $v_{0}+K x_{t}=u_{0 \mid t}^{o}+\delta u$ and $J\left(x_{t}, \mathbf{v}, \boldsymbol{\alpha}, \boldsymbol{\sigma}, \boldsymbol{\tau}\right) \leq J\left(x_{t}, \tilde{\mathbf{v}}_{t}, \tilde{\boldsymbol{\alpha}}_{t}, \tilde{\boldsymbol{\sigma}}_{t}, \tilde{\boldsymbol{\tau}}_{t}\right)$ as additional constraints (where $\tilde{\mathbf{v}}_{t}, \tilde{\boldsymbol{\alpha}}_{t}, \tilde{\boldsymbol{\sigma}}_{t}, \tilde{\boldsymbol{\tau}}_{t}$ are defined in (32) below).

(i) If the optimization in step 4(b) is infeasible, then implement $u_{t}=u_{0 \mid t}^{o}$.

(ii) Otherwise implement $u_{t}=u_{0 \mid t}^{o}+\delta u$.

Remark 15: The offline computation of $V, \lambda \in[0,1)$ and $K$ is feasible under the stabilizability condition of Assumption 3. A possible procedure is: (a) compute an ellipsoidal set, $\mathcal{E}$, that is robustly invariant for (1) with $\theta \in \Theta_{0}$ under linear feedback using semidefinite programming; (b) choose $V$ so that $\{x: V x \leq \mathbf{1}\}$ approximates $\mathcal{E}$ to a required degree of accuracy; (c) solve a linear program to determine the minimum $\lambda$ and the corresponding $K$ satisfying (4).

Remark 16: Step 4(b)(i) of the algorithm stipulates that, if the convex PE condition (30) cannot be met while at the same time robustly satisfying the constraints (2) and achieving a sufficiently rapid decrease in predicted cost, then robust constraint satisfaction takes priority over the PE condition.

\section{F. Recursive feasibility and closed loop stability}

Consider the suboptimal decision variables $\left(\tilde{\mathbf{v}}_{t}, \tilde{\boldsymbol{\alpha}}_{t}, \tilde{\boldsymbol{\sigma}}_{t}, \tilde{\boldsymbol{\tau}}_{t}\right)$ defined at time $t$ in terms of the optimal solution of (31) at time $t-1,\left(\mathbf{v}_{t-1}, \boldsymbol{\alpha}_{t-1}, \boldsymbol{\sigma}_{t-1}, \boldsymbol{\tau}_{t-1}\right)$, as

$$
\begin{aligned}
\tilde{\mathbf{v}}_{t} & =\left\{v_{1 \mid t-1}, \ldots, v_{N-1 \mid t-1}, 0\right\} \\
\tilde{\boldsymbol{\alpha}}_{t} & =\left\{\alpha_{1 \mid t-1}, \ldots, \alpha_{N \mid t-1}, \tilde{\alpha}_{N \mid t}\right\} \\
\tilde{\boldsymbol{\sigma}}_{t} & =\left\{\tilde{\sigma}_{0 \mid t}, \ldots, \tilde{\sigma}_{N-1 \mid t}, \tilde{\bar{\sigma}}_{N \mid t}\right\} \\
\tilde{\boldsymbol{\tau}}_{t} & =\left\{\tilde{\tau}_{0 \mid t}, \ldots, \tilde{\tau}_{N-1 \mid t}, \tilde{\bar{\tau}}_{N \mid t}\right\}
\end{aligned}
$$

where

$$
\begin{aligned}
\tilde{\sigma}_{k \mid t} & =\left[\max \left\{H_{Q} \alpha_{k+1 \mid t-1}\right\}-\bar{s}_{\infty}\left(\lambda_{t}\right)\right]_{\geq 0} \\
\tilde{\tau}_{k \mid t} & =\left[\max \left\{H_{R} \alpha_{k+1 \mid t-1}+R v_{k+1 \mid t-1}\right\}-\bar{t}_{\infty}\left(\lambda_{t}\right)\right]_{\geq 0}
\end{aligned}
$$

for all $k \in \mathbb{N}_{[0, N-1]}$, and

$$
\left[\tilde{\alpha}_{N \mid t}\right]_{i}=\max _{j \in\{1, \ldots, m\}} \theta_{t}^{(j)^{\top}} \hat{H}_{i} \alpha_{N \mid t-1}+\bar{w}_{i},
$$

for all $i \in \mathbb{N}_{\left[1, n_{\alpha}\right]}$, and

$$
\begin{aligned}
& \tilde{\bar{\sigma}}_{N \mid t}=\left[\frac{1}{1-\lambda_{t}}\left(\max \left\{H_{Q} \mathbf{1}\right\} \max \left\{\tilde{\alpha}_{N \mid t}\right\}-\bar{s}_{\infty}\left(\lambda_{t}\right)\right)\right]_{\geq 0} \\
& \tilde{\bar{\tau}}_{N \mid t}=\left[\frac{1}{1-\lambda_{t}}\left(\max \left\{H_{R} \mathbf{1}\right\} \max \left\{\tilde{\alpha}_{N \mid t}\right\}-\bar{t}_{\infty}\left(\lambda_{t}\right)\right)\right]_{\geq 0} .
\end{aligned}
$$

Proposition 17 (Recursive feasibility): Optimization (31) is feasible at all times $t \geq 1$ if it is feasible at $t=0$.

Lemma 18: If (31) is feasible at $t-1$, then at time $t$ :

$$
\tilde{\sigma}_{N-1 \mid t}+\tilde{\bar{\sigma}}_{N \mid t} \leq \bar{\sigma}_{N \mid t-1}
$$

$$
\begin{aligned}
& \quad+\max \left\{H_{Q} \mathbf{1}\right\}\left(\frac{1}{\left(1-\lambda_{t-1}\right)^{2}}-\frac{1}{\left(1-\lambda_{t}\right)^{2}}\right) \max \{\bar{w}\} \\
& \tilde{\tau}_{N-1 \mid t}+\tilde{\bar{\tau}}_{N \mid t} \leq \bar{\tau}_{N \mid t-1} \\
& \quad+\max \left\{H_{R} \mathbf{1}\right\}\left(\frac{1}{\left(1-\lambda_{t-1}\right)^{2}}-\frac{1}{\left(1-\lambda_{t}\right)^{2}}\right) \max \{\bar{w}\}
\end{aligned}
$$

where $\left(1-\lambda_{t-1}\right)^{-2} \geq\left(1-\lambda_{t}\right)^{-2} \geq 1$.

Proposition 19 (Asymptotic bounds on state and control): If the MPC optimization (31) is feasible at $t=0$, then the closed loop system satisfies the constraint $F x_{t}+G u_{t} \leq \mathbf{1}$ for all $t \in \mathbb{N}_{\geq 0}$ and the state and control input, $x_{t}$ and $u_{t}$, satisfy the asymptotic bounds:

$$
\begin{gathered}
\lim _{t \rightarrow \infty} \max \left\{Q x_{t}\right\} \leq \bar{s}_{\infty}\left(\lambda_{\infty}\right) \\
\lim _{t \rightarrow \infty} \max \left\{R u_{t}\right\} \leq \bar{t}_{\infty}\left(\lambda_{\infty}\right)
\end{gathered}
$$

where $\lambda_{\infty}=\lim _{t \rightarrow \infty} \lambda_{t}$.

\section{NUMERICAL EXAMPLE}

This section presents an example to illustrate the operation of the proposed adaptive robust MPC scheme. We first demonstrate the online parameter set estimation and constraint satisfaction.

Consider the second-order discrete-time uncertain linear system from [11], with model parameters

$$
\begin{gathered}
A_{0}=\left[\begin{array}{cc}
0.5 & 0.2 \\
-0.1 & 0.6
\end{array}\right], A_{1}=\left[\begin{array}{cc}
0.042 & 0 \\
0.072 & 0.03
\end{array}\right] \\
A_{2}=\left[\begin{array}{ll}
0.015 & 0.019 \\
0.009 & 0.035
\end{array}\right], A_{3}=\left[\begin{array}{ll}
0 & 0 \\
0 & 0
\end{array}\right] \\
B_{0}=\left[\begin{array}{c}
0 \\
0.5
\end{array}\right], B_{1}=\left[\begin{array}{l}
0 \\
0
\end{array}\right], B_{2}=\left[\begin{array}{l}
0 \\
0
\end{array}\right], B_{3}=\left[\begin{array}{c}
0.0397 \\
0.059
\end{array}\right]
\end{gathered}
$$

and with true system parameter $\theta^{*}=\left[\begin{array}{lll}0.8 & 0.2 & -0.5\end{array}\right]^{\top}$. The initial parameter set estimate is $\Theta_{0}=\left\{\theta:\|\theta\|_{\infty} \leq 1\right\}$. The elements of the disturbance sequence $\left\{w_{0}, w_{1}, \ldots\right\}$ are independent and identically (uniformly) distributed on $\mathbb{W}=$ $\left\{w \in \mathbb{R}^{2}:\|w\|_{\infty} \leq 0.1\right\}$. The state and input constraints are $\left[x_{t}\right]_{2} \geq 0.3$ and $u_{t} \leq 1$.

Simulations were performed in Matlab on a $2.7 \mathrm{GHz}$ Intel Core i5 processor. Algorithm 1 was solved using Yalmip and Gurobi. For $V$ with 9 rows and MPC prediction horizon $N=10$, the average solver time is $0.0127 \mathrm{~s}$, with a minimum $0.0098 \mathrm{~s}$ and maximum $0.0194 \mathrm{~s}$ over 5000 time steps. Figure 1 shows the cross-sections of the robust state tube predicted at $t=0$, with initial condition $x_{0}=(3,6)$. The state constraint $[x]_{2} \leq-0.3$ is robustly satisfied by the state tube predicted at $t=0$ and it can be seen that this constraint is satisfied by the closed loop state trajectory.

The change in the estimated parameter set over time is shown in Figure 2. Table I gives the parameter set volume, $\operatorname{vol}\left(\Theta_{t}\right)$ as a percentage of the initial volume $\operatorname{vol}\left(\Theta_{0}\right)$. The parameter set shrinks by $74 \%$ at the first time step and $87 \%$ by the 10th time step. After 5000 time steps, the parameter set is $0.0089 \%$ of its original size, implying that by this time it has been reduced almost to a point.

The reduction in the parameter set $\Theta_{t}$ causes a decrease in the optimal value of the MPC cost, $J^{o}\left(x_{t}\right)$, for the same 


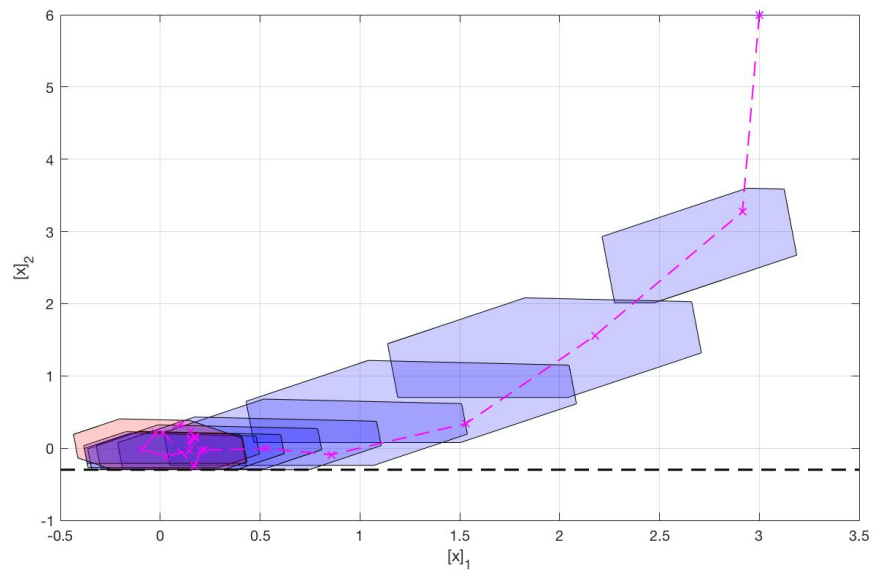

Fig. 1. Closed loop state trajectory (pink line) for initial condition $x_{0}=$ $(3,6)$, and predicted state tube cross-sections $\left\{\mathbb{X}_{0}, \ldots, \mathbb{X}_{N}\right\}$ at time $t=0$, with $\mathbb{X}_{N}$ shown in red.
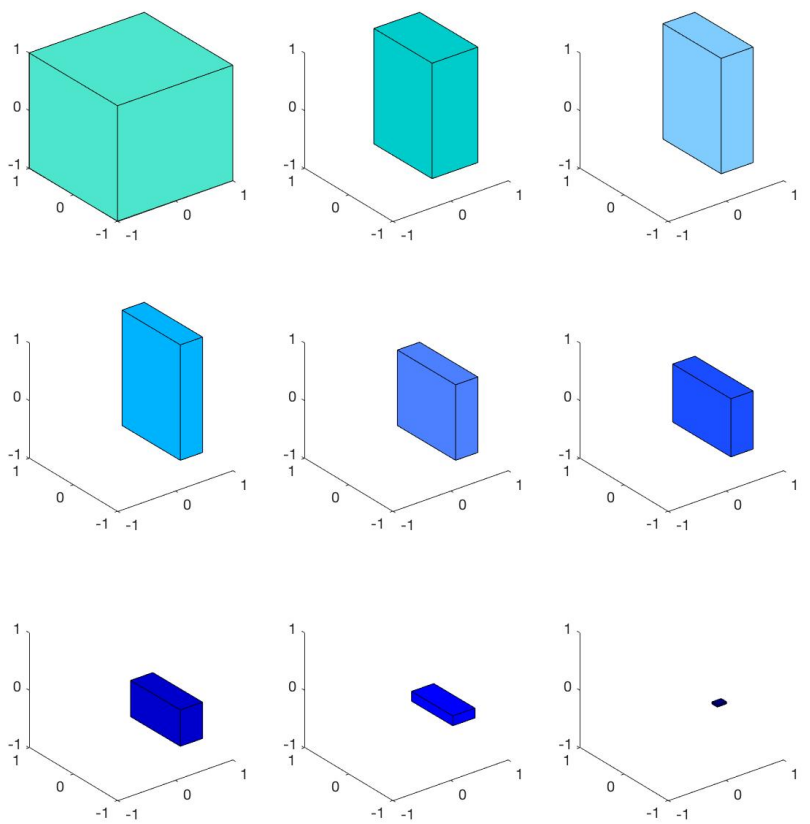

Fig. 2. Parameter set $\Theta_{t}$ at time steps $t=0,1,2$ (1st row) $t=10,25,50$ (2nd row) $t=100,500,5000$ (3rd row)

TABLE I. Volume of parameter set $\Theta_{t}$ as a percentage of $\Theta_{0}$

\begin{tabular}{lcccccccc}
\hline Time step $t$ & 0 & 1 & 2 & 10 & 25 & 50 & 100 & 500 \\
Volume (\%) & 100 & 26.1 & 18.3 & 12.7 & 8.3 & 6.3 & 3.4 & 0.7 \\
\hline
\end{tabular}

value of the state $x_{t}$ and the same set of constraints. The initial parameter set $\Theta_{0}$ gives an optimal predicted cost of $J^{o}(x)=62.2$ for $x=x_{0}=(3,6)$, whereas with $\Theta_{500}$ the optimal cost is $J^{o}(x)=57.9$ (6.9\% reduction), and with $\Theta_{5000}$ the optimal cost is $J^{o}(x)=53.9$ ( $13.3 \%$ reduction), and with no parameter uncertainty $\left(\Theta=\theta^{*}\right)$ the optimal cost is $J^{o}(x)=52.70$ ( $15.3 \%$ reduction $)$.

\section{CONCLUSions}

An adaptive robust MPC algorithm that combines robust tube MPC and set membership identification has been proposed. A min-max cost function and tube MPC formulation guarantee robust constraint satisfaction for the closed loop system. The algorithm is proven to be recursive feasible and the state is shown to converge asymptotically to a set that depends explicitly on the uncertainty in model parameters. The uncertain parameter set $\Theta$ is zonotopic, which reduces the computational load while allowing sufficient flexibility. The offline and online optimizations are linear programming problems. Conditions for persistent excitation are linearized and included whenever possible in the MPC optimization to enforce parameter set convergence. A numerical example illustrates that the algorithm is able to reduce the parameter set size while satisfying the constraints robustly and steering the closed loop state to a neighbourhood of the origin.

Future work will consider more general zonotopic forms for the uncertain parameter set $\Theta$ that retain fixed complexity and tractable conversion from $\mathrm{H}$ - to $\mathrm{V}$-form. In addition, convex PE conditions that apply to future predicted trajectories will be investigated in order to ensure persistency of excitation whenever this is feasible.

\section{REFERENCES}

[1] D. Q. Mayne, J. Rawling, C. Rao, and P. Scokaert, "Constrained model predictive control: stability and optimality," Automatica, vol. 36, pp. 789-814, 2000.

[2] B. Kouvaritakis and M. Cannon, Model Predictive Control: Classical, Robust and Stochastic. Springer, London, 2015.

[3] G. Tao, "Multivariable adaptive control: A survey," Automatica, vol. 50, no. 11, pp. 2737-2764, 2014.

[4] D. Q. Mayne, "Model predictive control: Recent developments and future promise," Automatica, vol. 50, no. 12, pp. 2967-2986, 2014.

[5] S. Qin and T. Badgwell, "A survey of industrial model predictive control technology," Control Engineering Practice 11, vol. 11, pp. $733-764,2003$.

[6] A. Aswani, H. Gonzalez, S. S. Sastry, and C. Tomlin, "Provably safe and robust learning-based model predictive control," Automatica, vol. 49, no. 5, pp. 1216-1226, 2013.

[7] M. Tanaskovic, L. Fagiano, R. Smith, and M. Morari, "Adaptive receding horizon control for constrained MIMO systems," Automatica, vol. 50, no. 12, pp. 3019-3029, 2014.

[8] T. A. N. Heirung, B. E. Ydstie, and B. Foss, "An MPC approach to dual control," IFAC Proceedings Volumes (IFAC-PapersOnline), vol. 10, no. PART 1, pp. 69-74, 2013.

[9] V. A. Akpan and G. D. Hassapis, "Nonlinear model identification and adaptive model predictive control using neural networks," ISA Transactions, vol. 50, no. 2, pp. 177-194, 2011.

[10] B. M. Reese and E. G. Collins, "A graph search and neural network approach to adaptive nonlinear model predictive control," Engineering Applications of Artificial Intelligence, vol. 55, pp. 250-268, 2016.

[11] M. Lorenzen, F. Allgöwer, and M. Cannon, "Adaptive Model Predictive Control with Robust Constraint Satisfaction," IFAC-PapersOnLine, vol. 50, no. 1, pp. 3313-3318, 2017.

[12] S. V. Raković, B. Kouvaritakis, R. Findeisen, and M. Cannon, "Homothetic tube model predictive control," Automatica, vol. 48, no. 8, pp. 1631-1638, 2012.

[13] J. Fleming, B. Kouvaritakis, and M. Cannon, "Robust Tube MPC for Linear Systems with Multiplicative Uncertainty," IEEE Transactions on Automatic Control, vol. 60, no. 4, pp. 1087-1092, 2015.

[14] G. Marafioti, R. R. Bitmead, and M. Hovd, "Persistently exciting model predictive control," International Journal of Adaptive Control and Signal Processing, vol. 28, no. 6, pp. 536-552, 2014.

[15] L. Chisci, A. Garulli, A. Vicino, and G. Zappa, "Block recursive parallelotopic bounding in set membership identification," Automatica, vol. 34, no. 1, pp. 15-22, 1998.

[16] S. M. Veres, H. Messaoud, and J. P. Norton, "Limited-complexity model-unfalsifying adaptive tracking-control," International Journal of Control, vol. 72, no. 15, pp. 1417-1426, 1999.

[17] F. Blanchini and S. Miani, Set-Theoretic Methods in Control. Birkhauser Boston, 2008.

[18] E. R. Bai, H. Cho, and R. Tempo, "Convergence Properties of the Membership Set," Automatica, vol. 34, no. 10, pp. 1245-1249, 1998. 


\section{APPENDIX A: PROOFS OF MAIN RESULTS}

\section{Proof of Lemma 7:}

The constraint (2) applied to $x_{k} \in \mathbb{X}_{k}$ and $u_{k}=K x_{k}+v_{k}$ is equivalent to

$$
(F+G K) x+G v_{k} \leq \mathbf{1} \text { for all } x \in \mathbb{X}_{k},
$$

and since $\mathbb{X}_{k}=\left\{x: V x \leq \alpha_{k}\right\}$, this can be expressed

$$
\left\{x: V x \leq \alpha_{k}\right\} \subseteq\left\{x:(F+G K) x \leq \mathbf{1}-G v_{k}\right\} .
$$

From [17], condition (42) is therefore equivalent to the existence of $H_{c}$ satisfying:

$$
\begin{aligned}
& H_{c} \geq 0 \\
& H_{c} V=F+G K \\
& H_{c} \alpha_{k}+G v_{k} \leq \mathbf{1}
\end{aligned}
$$

In the robust MPC implementation, the predicted control sequence $v_{0}, \ldots, v_{N-1}$ and state tube variables $\alpha_{1}, \ldots, \alpha_{N}$ are optimized online. To ensure that the online MPC optimization is convex, matrices $H_{c}$ satisfying (43a) and (43b) are computed offline. This is done by solving (9) for each row of $H_{c}$, so that (8) (which is linear in the online decision variables) is sufficient to ensure (42).

\section{Proof of Lemma 11:}

If $V x_{k} \leq \alpha_{k}$, then $V x_{k+1} \leq \alpha_{k+1}$ where, from (4), the maximum element of $\alpha_{k+1}$ satisfies, for all $k \geq N$,

$$
\begin{aligned}
\max \left\{\alpha_{k+1}\right\} & =\max _{\theta \in \Theta, x_{k} \in \mathbb{X}_{k}} \max \left\{V \Phi(\theta) x_{k}+\bar{w}\right\} \\
& \leq \max _{\theta \in \Theta, x_{k} \in \mathbb{X}_{k}} \max \left\{V \Phi(\theta) x_{k}\right\}+\max \{\bar{w}\} \\
& \leq \lambda \max \left\{\alpha_{k}\right\}+\max \{\bar{w}\},
\end{aligned}
$$

and hence, for all $k \geq N$,

$$
\max \left\{\alpha_{k}\right\} \leq \lambda^{k-N} \max \left\{\alpha_{N}\right\}+\frac{1-\lambda^{k-N}}{1-\lambda} \max \{\bar{w}\} .
$$

Defining $s_{k}=\max \left\{H_{Q} \alpha_{k}\right\}$ and $t_{k}=\max \left\{H_{R} \alpha_{k}\right\}$ for all $k \geq N$, the bounds (20) and (21) then follow from $\max \left\{H_{Q} \alpha_{k}\right\} \leq \max \left\{H_{Q} \mathbf{1}\right\} \max \left\{\alpha_{k}\right\}, \max \left\{H_{R} \alpha_{k}\right\} \leq$ $\max \left\{H_{R} \mathbf{1}\right\} \max \left\{\alpha_{k}\right\}$ and Lemma 10.

Proof of Lemma 12:

From the definition of $\bar{s}_{k}(\lambda)$ in (20) we have

$$
\begin{aligned}
& \sum_{k=N}^{\infty}\left[\max _{x \in \mathbb{X}_{k}} \max \{Q x\}-\bar{s}_{\infty}(\lambda)\right]_{\geq 0} \leq \sum_{k=N}^{\infty}\left[\bar{s}_{k}(\lambda)-\bar{s}_{\infty}(\lambda)\right]_{\geq 0} \\
& =\left[\max \left\{H_{Q} \mathbf{1}\right\} \frac{1}{1-\lambda}\left(\max \left\{\alpha_{N}\right\}-\frac{1}{1-\lambda} \max \{\bar{w}\}\right)\right]_{\geq 0}=\bar{\sigma}_{N} .
\end{aligned}
$$

A similar argument demonstrates the bound $\bar{\tau}_{N}$.

\section{Proof of Proposition 17:}

If the optimization (31) is feasible at time $t-1$, then $\left(\tilde{\mathbf{v}}_{t}, \tilde{\boldsymbol{\alpha}}_{t}, \tilde{\boldsymbol{\sigma}}_{t}, \tilde{\boldsymbol{\tau}}_{t}\right)$ is:

- feasible at time $t$ for (8), (10) for $k \in \mathbb{N}_{0, N-2]}$ due to feasibility of (8), (10) at $t-1$ and $\Theta_{t} \subseteq \Theta_{t-1}$;

- feasible at time $t$ for (8), (10) for $k=N-1$ due to feasibility of (13), (14) at $t-1$ and $\Theta_{t} \subseteq \Theta_{t-1}$;
- feasible at time $t$ for (25), (26) for $k \in \mathbb{N}_{[0, N-1]}$ because of the definitions (33), (34) of $\tilde{\sigma}_{k \mid t}, \tilde{\tau}_{k \mid t}$;

- feasible at time $t$ for (12) because $V x_{t} \leq \alpha_{1 \mid t-1}$;

- feasible at time $t$ for (13), since feasibility of (13) at $t-1$ and the definition (35) of $\tilde{\alpha}_{N \mid t}$ together imply that $\alpha_{N \mid t-1} \geq \tilde{\alpha}_{N \mid t}$ due to $\Theta_{t} \subseteq \Theta_{t-1}$

- feasible at time $t$ for (14) since the constraint is by assumption feasible at $t-1$ and $\tilde{\alpha}_{N \mid t} \leq \alpha_{N \mid t-1}$;

- feasible at time $t$ for (27), (28) because of the definitions (36), (37) of $\tilde{\bar{\sigma}}_{N \mid t}, \tilde{\bar{\tau}}_{N \mid t}$.

It follows that $\left(\tilde{\mathbf{v}}_{t}, \tilde{\boldsymbol{\alpha}}_{t}, \tilde{\boldsymbol{\sigma}}_{t}, \tilde{\boldsymbol{\tau}}_{t}\right)$ is a feasible (possibly suboptimal) solution of (31) at time $t$.

\section{Proof of Lemma 18:}

The definitions of $\tilde{\alpha}_{N \mid t}$ in (35) and $\lambda_{t}$ in Algorithm 1 imply

$$
\max \left\{\tilde{\alpha}_{N \mid t}\right\} \leq \lambda_{t} \max \left\{\alpha_{N \mid t-1}\right\}+\max \{\bar{w}\} .
$$

From (36) and (33) we therefore obtain

$\tilde{\bar{\sigma}}_{N \mid t} \leq\left[\max \left\{H_{Q} 1\right\}_{1-\lambda_{t}} \frac{\lambda_{t}}{1-\lambda_{t}}\left(\max \left\{\alpha_{N \mid t-1}\right\}-\frac{1}{1-\lambda_{t}} \max \{\bar{w}\}\right)\right]_{\geq 0}$ $\tilde{\sigma}_{N-1 \mid t} \leq\left[\max \left\{H_{Q} \mathbf{1}\right\}\left(\max \left\{\alpha_{N \mid t-1}\right\}-\frac{1}{1-\lambda_{t}} \max \{\bar{w}\}\right)\right]_{\geq 0}$.

But $\lambda_{t} \leq \lambda_{t-1}$ since $\Theta_{t} \subseteq \Theta_{t-1}$ and it follows that

$\tilde{\sigma}_{N-1 \mid t}+\tilde{\bar{\sigma}}_{N \mid t}$

$\leq\left[\max \left\{H_{Q} \mathbf{1}\right\}\left(\frac{1}{1-\lambda_{t-1}} \max \left\{\alpha_{N \mid t-1}\right\}-\frac{1}{\left(1-\lambda_{t}\right)^{2}} \max \{\bar{w}\}\right)\right]_{\geq 0}$

$=\bar{\sigma}_{N \mid t-1}+\max \left\{H_{Q} \mathbf{1}\right\}\left(\frac{1}{\left(1-\lambda_{t-1}\right)^{2}}-\frac{1}{\left(1-\lambda_{t}\right)^{2}}\right) \max \{\bar{w}\}$,

which demonstrates (38). The bound in (39) can be demonstrated using a similar argument, whereas $\left(1-\lambda_{t-1}\right)^{-2} \geq$ $\left(1-\lambda_{t}\right)^{-2} \geq 1$ follows from $\lambda_{t-1} \geq \lambda_{t} \geq 0$.

Proof of Proposition 19:

First note that $\lambda_{t}$ necessarily converges to a limit $\lambda_{\infty} \leq \lambda_{0}$, since by assumption $\Theta_{0}$ is compact and $\Theta_{t} \subseteq \Theta_{t-1}$ for all $t$. To simplify notation, we use $J_{t}^{o}$ to denote the optimal value, $J^{o}\left(x_{t}\right)=J\left(x_{t}, \mathbf{v}_{t}, \boldsymbol{\alpha}_{t}, \boldsymbol{\sigma}_{t}, \boldsymbol{\tau}_{t}\right)$, of the MPC cost (31). Then from Proposition 17 and Lemma 18 we have

$$
\begin{aligned}
J_{t}^{o} \leq J\left(x_{t}, \tilde{\mathbf{v}}_{t}, \tilde{\boldsymbol{\alpha}}_{t}, \tilde{\boldsymbol{\sigma}}_{t}, \tilde{\boldsymbol{\tau}}_{t}\right) & \\
\leq J_{t-1}^{o}- & {\left[\max \left\{Q x_{t-1}\right\}-\bar{s}_{\infty}\left(\lambda_{t-1}\right)\right]_{\geq 0} } \\
- & {\left[\max \left\{R u_{t-1}\right\}-\bar{t}_{\infty}\left(\lambda_{t-1}\right)\right]_{\geq 0} } \\
+ & \left(\max \left\{H_{Q} \mathbf{1}\right\}+\max \left\{H_{R} \mathbf{1}\right\}\right) \\
& \left(\frac{1}{\left(1-\lambda_{t-1}\right)^{2}}-\frac{1}{\left(1-\lambda_{t}\right)^{2}}\right) \max \{\bar{w}\} .
\end{aligned}
$$

For any positive integer $T$ we therefore obtain

$$
\begin{gathered}
\sum_{t=0}^{T-1}\left[\max \left\{Q x_{t}\right\}-\bar{s}_{\infty}\left(\lambda_{t}\right)\right]_{\geq 0}+\left[\max \left\{R u_{t}\right\}-\bar{t}_{\infty}\left(\lambda_{t}\right)\right]_{\geq 0} \\
\leq J_{0}^{o}-J_{T}^{o}+\left(\max \left\{H_{Q} \mathbf{1}\right\}+\max \left\{H_{R} \mathbf{1}\right\}\right) \\
\left(\frac{1}{\left(1-\lambda_{0}\right)^{2}}-1\right) \max \{\bar{w}\} .
\end{gathered}
$$

The RHS of this inequality is finite since $J_{T}^{o} \geq 0$ for all $T$. It follows that the sum on the LHS converges as $T \rightarrow \infty$, which implies the asymptotic bounds in (40) and (41). 